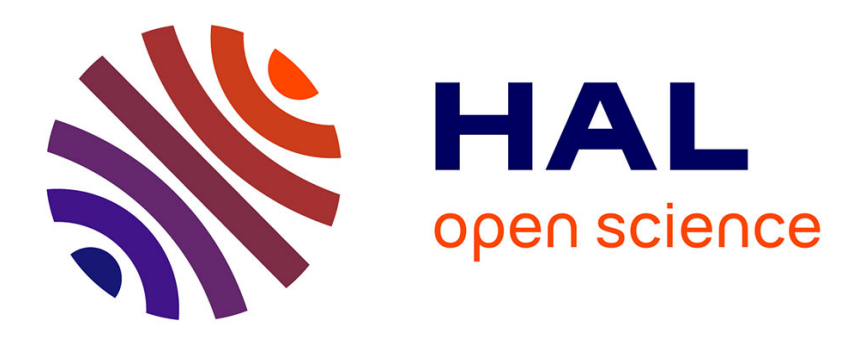

\title{
Spin Fluctuations beyond the Gutzwiller Approximation: a Renormalised Paramagnon Theory
}

R Raimondi, M. Lavagna

\section{To cite this version:}

R Raimondi, M. Lavagna. Spin Fluctuations beyond the Gutzwiller Approximation: a Renormalised Paramagnon Theory. Journal of Low Temperature Physics, 1995, 99 (3-4), pp.355 - 357. 10.1007/BF00752308 . hal-01896235

\section{HAL Id: hal-01896235 https://hal.science/hal-01896235}

Submitted on 17 Oct 2018

HAL is a multi-disciplinary open access archive for the deposit and dissemination of scientific research documents, whether they are published or not. The documents may come from teaching and research institutions in France or abroad, or from public or private research centers.
L'archive ouverte pluridisciplinaire HAL, est destinée au dépôt et à la diffusion de documents scientifiques de niveau recherche, publiés ou non, émanant des établissements d'enseignement et de recherche français ou étrangers, des laboratoires publics ou privés. 


\title{
Spin Fluctuations beyond the Gutzwiller Approximation : a Renormalised Paramagnon Theory
}

\author{
R.Raimondi* and M.Lavagna \\ Centre d'Etudes Nucléaires de Grenoble, DRFMC-SPSMS, 17 Rue des Martyrs, 38054 \\ Grenoble Cedex 9, France
}

We analyze the spin fluctuations in the single-band lattice Hubbard model by using the slave-boson technique. It is shown that, at the Gaussian level beyond the saddle-point approximation, spin fluctuations obey a simple RPA-like form at all frequencies and wavevectors. Spin fluctuations, then, appear to give rise to an effective renormalized paramagnon theory opening new perspectives for the discussion of the effect of a magnetic field on Mott-Hubbard systems.

PACS numbers: $71.30+h, 75.30 \mathrm{Kz}, 71.28+d, 72.80 \mathrm{Ga}$

Spin fluctuations in a paramagnetic metal have been an intensive subject of research for many years. Stoner and paramagnon theories are among the most used in interpreting experiments in a large variety of circumstances because of their simplicity and direct physical interpretation. For example, the recently measured magnetization of liquid ${ }^{3} \mathrm{He}^{1}$ as a function of the magnetic field, bending downwards below the initial slope, appears to be explained, at least qualitatively, by a paramagnon model. ${ }^{2}$ This should be contrasted with the Gutzwiller approximation for the single-band Hubbard model which predicts a metamagnetic transition at high fields ${ }^{3}$ above a critical value of the interaction. More recently, the infinite dimension approach $^{4}$ brought new enlightenments on the question, confirming the Gutzwiller approximation for small enough value of the interaction, but predicting a smooth transition at large $U$ from paramagnetic Mott insulator to fully-polarized insulator without any evidence for metamagnetism.

In this paper, we address the problem of accounting for spin fluctuations in the Hubbard model beyond the Gutzwiller approximation. The analysis is performed within the four slave-boson approach introduced by Kotliar and Ruckenstein. ${ }^{5}$ The slave-boson approach at its lowest level of approximation (saddle-point) is equivalent to the Gutzwiller approximation and allows for a systematic expansion. The first correction, i.e. Gaussian fluctuations about saddle-point, has been considered by a number of authors, ${ }^{6}$ although much of their analysis has been confined to the small 
frequency and wavelength limit. Our main aim, here, is to extend this analysis for arbitrary wavevector and frequency. It will be shown that the expression for the spin fluctuation spectrum has a simple RPA form as in the paramagnon theory, with particle mass and local interaction suitably renormalized. This result suggests that the metamagnetic transition which has been seen as a consequence of the strong localized character of the quasiparticles close to a Mott metal-insulator transition, could be reduced by allowing spin fluctuations, as in the paramagnon theory.

Our starting point is the formulation of the single-band Hubbard model in the slave-boson language, ${ }^{5}$ whose Lagrangian reads :

$$
\begin{aligned}
\mathcal{L}(\tau) & =-t \sum_{i, j, \sigma} z_{i \sigma}^{\dagger} z_{j \sigma} c_{i \sigma}^{\dagger} c_{j \sigma}+\sum_{i, \sigma} c_{i \sigma}^{\dagger}\left(\partial / \partial \tau+\lambda_{i \sigma}^{(2)}-\mu\right) c_{i \sigma} \\
& +\sum_{i} d_{i}^{\dagger}\left(\partial / \partial \tau+\lambda_{i}^{(1)}+U-\sum_{\sigma} \lambda_{i \sigma}^{(2)}\right) d_{i}+\sum_{i} e_{i}^{\dagger}\left(\partial / \partial \tau+\lambda_{i}^{(1)}\right) e_{i} \\
& +\sum_{i, \sigma} p_{i \sigma}^{\dagger}\left(\partial / \partial \tau+\lambda_{i}^{(1)}-\lambda_{i \sigma}^{(2)}\right) p_{i \sigma}-\sum_{i} \lambda_{i}^{(1)}
\end{aligned}
$$

The physical subspace is defined by the constraints : $e_{i}^{\dagger} e_{i}+d_{i}^{\dagger} d_{i}+\sum_{\sigma} p_{i \sigma}^{\dagger} p_{i \sigma}=1$ and $d_{i}^{\dagger} d_{i}+p_{i \sigma}^{\dagger} p_{i \sigma}=c_{i \sigma}^{\dagger} c_{i \sigma}$. The $z_{i \sigma}$ are composite fields of the form $z_{i \sigma}=U_{i}\left(e_{i}^{\dagger} p_{i \sigma}+\right.$ $\left.p_{i-\sigma}^{\dagger} d_{i}\right) V_{i}$ in which $U_{i}$ and $V_{i}$ are kept unspecified at that level. Because charge and spin fluctuations decouple at the Gaussian level, it is convenient to introduce the following symmetric and antisymetric combinations $p_{q s(a)}=\frac{1}{\sqrt{2}}\left(p_{q \uparrow} \pm p_{q \downarrow}\right)$. Considering spin fluctuations is equivalent to study the fluctuations of the field $p_{q a}$. The corresponding Lagrangian is given by $\mathcal{L}=\frac{1}{2} \sum_{q \nu} x_{\alpha}^{\dagger}(q, \nu) \Gamma_{\alpha, \beta}(q, \nu) x_{\beta}(q, \nu)$ where $x_{\alpha}(q, \nu)=\left(p_{q \nu a}, p_{-q-\nu a}^{\dagger}\right)$ and :

$$
\Gamma(q, \nu)=\left(\begin{array}{cc}
i \nu+\lambda_{0}^{(1)}-\lambda_{0}^{(2)}-\Sigma_{11}(q, \nu) & -\Sigma_{12}(q, \nu) \\
-\Sigma_{21}(q, \nu) & -i \nu+\lambda_{0}^{(1)}-\lambda_{0}^{(2)}-\Sigma_{22}(q, \nu)
\end{array}\right)
$$

having indicated with $\Sigma_{\alpha \beta}(q, \nu)$ the matrix of the self-energies written as:

$$
\begin{gathered}
\Sigma_{\alpha \beta}(q, \nu)=-4\left[(\alpha, \beta)+\frac{1}{2}(\alpha)(\beta)^{\dagger}+\frac{1}{2}(\alpha)^{\dagger}(\beta)\right] S_{0}+2(\alpha)(\beta)^{\dagger} i \nu P(q, \nu) \\
-2(\alpha)^{\dagger}(\beta) i \nu P(-q,-\nu)+2\left[(\alpha)^{\dagger}(\beta)^{\dagger}+(\alpha)(\beta)+(\alpha)^{\dagger}(\beta)+(\alpha)(\beta)^{\dagger}\right] \Pi(q, \nu)
\end{gathered}
$$

where we have adopted the convenient notation $(\alpha) \equiv \frac{\partial z_{\sigma}}{\partial x_{\alpha}},(\alpha)^{\dagger} \equiv \frac{\partial z_{\sigma}^{\dagger}}{\partial x_{\alpha}},(\alpha, \beta) \equiv$ $\frac{1}{2} z_{0} \frac{\partial^{2}}{\partial x_{\alpha} \partial x_{\beta}}\left(z_{\sigma}+z_{\sigma}^{\dagger}\right)$. The various quantities appearing in Eq. $(3)$ are given by $S_{0}=$ $\sum_{k}\left(-2 t \epsilon_{k}\right) f\left(-2 t z_{0}^{2} \epsilon_{k}\right), \Pi(q, \nu)=\left(2 t z_{0}\right)^{2} \sum_{k, n} \epsilon_{k+q} \epsilon_{k} G(k, n) G(k+q, n+\nu)$, and $P(q, \nu)=2 t \sum_{k, n} \epsilon_{k} G(k, n) G(k+q, n+\nu)$ with $G(k, n)$ being the Green function of the saddle-point paramagnetic solution $G(k, n)=\left(i \omega_{n}+2 t z_{0}^{2} \epsilon_{k}\right)^{-1}$. To analyse 
the spin fluctuations, we take the determinant of the fluctuation matrix $\Gamma(q, \nu)$ :

$$
\begin{gathered}
\operatorname{det} \Gamma(q, \nu)=\nu^{2}+\left(\lambda_{0}^{(1)}-\lambda_{0}^{(2)}-4\left[\left(p_{a}^{\dagger}, p_{a}\right)+\left(p_{a}, p_{a}\right)\right] S_{0}\right) \\
\left(\lambda_{0}^{(1)}-\lambda_{0}^{(2)}-4\left[\left(p_{a}^{\dagger}, p_{a}\right)-\left(p_{a}, p_{a}\right)+2\left(p_{a}\right)^{2}\right] S_{0}-4\left(p_{a}\right)^{2} \frac{\nu^{2}}{z_{0}^{2}} \chi(q, \nu)\right)
\end{gathered}
$$

having used the fact that $i \nu[P(q, \nu)-P(-q,-\nu)]=-\nu^{2} / z_{0}^{2} \chi(q, \nu), \chi$ being the Lindhardt function for the quasi-particles. The expressions derived up to this point are general. Let us now specify them to the cases of Regime I ( $\delta=0$ and $U<U_{c}$ ) and Regime II $\left(\delta<<1\right.$ and $\left.U>U_{c}\right)$ as defined in Ref. ${ }^{6}$ We use at that step the fields $z_{i \sigma}$ corresponding to the Kotliar-Ruckenstein choice ${ }^{5}$ which allows to recover the uncorrelated limit $(U=0)$ at saddle-point level. It is easy, but tedious, to see that $\mathrm{Eq} .(4)$ can be rewritten in the final more transparent form :

$$
\operatorname{det} \Gamma(q, \nu)=\nu^{2}\left\{1-F_{0}^{a}\left(z_{0}^{2} / \rho_{F}^{0}\right) \chi(q, \nu)\right\}
$$

with $\lim _{\nu=0, q \rightarrow 0} \chi=-\rho_{F}^{0} / z_{0}^{2}, \rho_{F}^{0}$ being the free particle density of states. $F_{0}^{a}$ is the Landau paramater, as obtained in Refs. ${ }^{3},{ }^{6}$ playing the role of the local effective interaction whatever the value of the coupling is, and equalling $-U$ in the weakcoupling limit giving back the paramagnon theory.

Eq.(5) is now essential for our discussion of the effect of a magnetic field on Mott-Hubbard systems. It means that the free energy of the system is given by the sum of two contributions : first, the saddle-point term totally equivalent to the Gutzwiller approximation, and second the spin-fluctuation term exactly as in the paramagnon theory but with renormalized values of the parameters $\frac{m^{*}}{m}$ and $F_{0}^{a}$. It reconciliates the "almost-localized" picture provided by the G.A. and the "almost-magnetic" one given by the paramagnon theory. By allowing self-consistent effects in presence of an external magnetic field, it appears extremely interesting to examine to what extent spin-fluctuations will draw the system away from the metamagnetic transition predicted by the Gutwiller approximation. Such study is presently under investigation.

*Present address : School of Physics ans Materials, Lancaster University, LA1 4 YB, U.K.

\section{ACKNOWLEDGMENTS}

This work was supported by E.U. contract number ERB 4050 PL920925.

\section{REFERENCES}

1. S.A.J.Wiegers, P.E.Wolf, and L.Puech Phys. Rev. Lett. 66,2895 (1991).

2. M.T.Béal-Monod and E.Daniel Phys. Rev. B27, 4467 (1983); Phys. Rev. Lett.68,3817 (1992).

3. D.Vollhardt Rev. Mod. Phys.56, 99 (1984).

4. L.Laloux, A.Georges, and W.Krauth Phys. Rev. B50, 3092 (1994).

5. G.Kotliar and A.Ruckenstein Phys. Rev. Lett.57, 1362 (1986).

6. See M.Lavagna Phys. Rev. B41, 142(1990) and ref. therein. 